\title{
A case of migration of fractured tracheostomy tube-a case presentation
}

\author{
Gaurang Singhal, Pallika Kumar ${ }^{*}$ (D), Sachin Goel, Sanjeet Goit and Anjali Pathak
}

\begin{abstract}
Background: Tracheostomy is widely used for securing the airway; however, complications do occur. One such rare complication is a fracture of the tracheostomy tube which presents as a foreign body of the tracheobronchial tree.

Case presentation: We report a case of a 7 -year-old child who presented with mild respiratory distress following fracture and migration of non-metallic tracheostomy tube in the airway. Successful removal was done via rigid bronchoscopy under general anaesthesia.

Conclusion: Careful inspection of the tracheostomy tube before placing and appropriate tracheostomy care is the cornerstone to prevent such rare complications associated with a tracheostomy.
\end{abstract}

Keywords: Foreign body, Tracheostomy tube, Bronchoscopy

\section{Background}

Tracheostomy is a common procedure undertaken to secure the airway as an emergency procedure or in patients requiring prolonged ventilation $[1,2]$. Tracheostomy tubes can either be metallic or non-metallic. About $20 \%$ of the patients who are tracheostomised are discharged with tracheostomy in situ [3, 4]. Thus these patients require proper care and management and inappropriate care may lead to unexpected complications. Fracture of the tracheostomy tube and its migration is one such rare complication. The most commonly known risk factors are the usage of the same tracheostomy tube, alkaline bronchial secretions, repeated cleaning and sterilization, ageing of the tracheostomy tube, tissue reaction to the tube and manufacturing defects [5-10]. Thus, there is a need to understand this particular complication for prevention, early recognition and management.

*Correspondence: pallikakumar@gmail.com

Department of Otorhinolaryngology ABVIMS \& Dr RML Hospital, New Delhi, India

\section{Case presentation}

A 7-year-old child presented in emergency with a history of aspiration of a part of the tracheostomy tube while doing the suctioning of the tube by the parents at their home. They noticed that neck plate was present at the place while the tube was missing.

The child was diagnosed as the case of neurodegenerative disorder with lung collapse and recurrent pneumonia 10 months back for which the patient was tracheostomised 10 months back. The patient was on a decannulation trial and had his tracheostomy tube changed 1 day before in the otolaryngology outpatient department to a $4.5-\mathrm{mm}$ uncuffed tracheostomy tube polyvinyl chloride type.

At the time of presentation, the patient was haemodynamically stable with tachypnoea $(25 / \mathrm{min})$ and tachycardia $(100 / \mathrm{min})$. The saturation of the patient was $90 \%$ on room air but was maintaining saturation of $99 \%$ with an oxygen face mask. Neck examination revealed patent and contracted stoma site with minimal granulations around. Air entry was reduced on the left side as compared to the right side. The patient was then subjected to a chest $\mathrm{X}$-ray (postero-anterior view) which revealed part of the tracheostomy tube in the left main bronchus (Fig. 1). The airway of the patient was then secured with $4.5 \mathrm{~mm}$ 


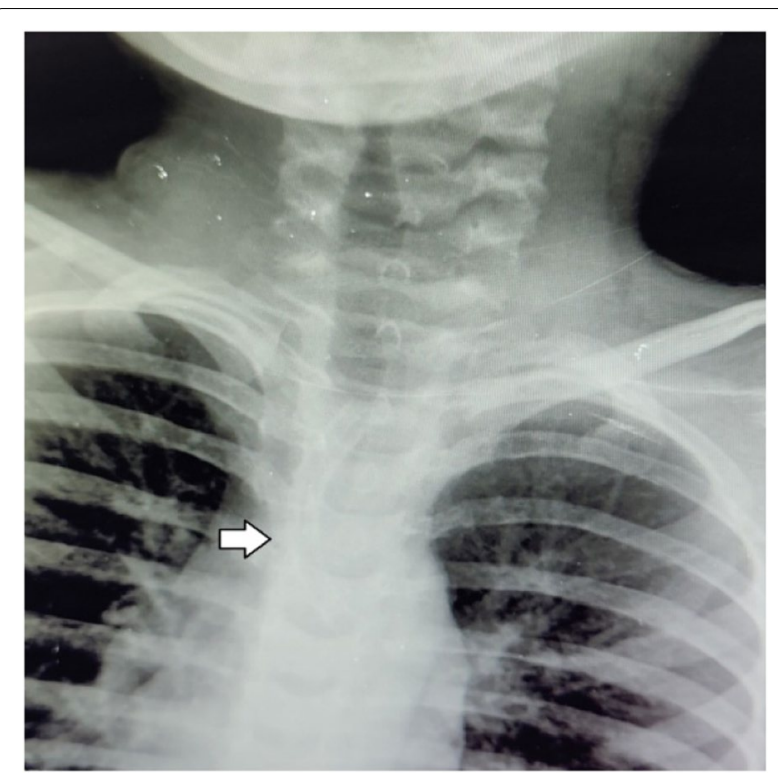

Fig. 1 Xray soft tissue neck-AP view arrow showing part of tracheostomy tube

tracheostomy tube polyvinyl chloride type with oxygen inhalation before shifting to the operation theatre.

Rigid bronchoscopy was done with a size 4 bronchoscope while the oxygenation was done through the tracheostomy tube. Upon visualization of the neck of the tracheostomy tube, the cuff was deflated and bronchoscope was advanced in the trachea beside the tube with ventilation continued. Fractured tracheostomy was visualized around $3 \mathrm{~cm}$ below the stoma and was grasped with optical forceps and was delivered out along with the bronchoscope in toto (Fig. 2) and immediately the airway was secured with polyvinyl chloride $4.5 \mathrm{~mm}$ tracheostomy tube and ventilation resumed. The child was shifted to the paediatric intensive care unit for observation and was discharged 2 days after the procedure and is doing well on follow-up.

\section{Discussion}

Bassoe and Boe were the first to report aspiration of fractured tracheostomy tube in 1960, [11] since then various similar cases have been reported in the literature.

Metallic tracheostomy tubes are usually preferred in older children and those requiring prolonged tracheostomy, however in younger children non metallic tubes are preferred due to less airway resistance, as seen in metallic tubes due to usage of inner tubes, and they are larger, more pliable and snuggly fit the airway. Additionally, mucus adherence is less due to the inert nature and smooth surface of non-metallic tubes. However non-metallic tubes are costlier and require regular

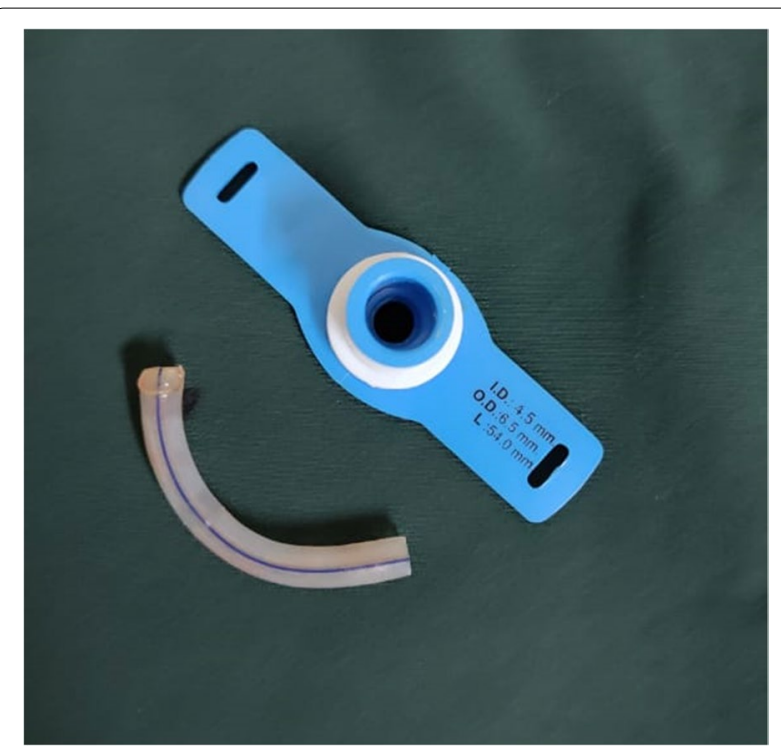

Fig. 2 Broken tracheostomy tube

suctioning [12]. Review done by Piromachai et al. on 20 cases concluded that fracture of the metallic tube was more common as compared to the non-metallic tracheostomy tubes and trachea and the right main bronchus was the most common dislodgement site [2]. Similar findings were also reported by Parida et al. in their review of 8 cases [7]. In our case, the tube was polyvinyl chloride type and was dislodged in the trachea and left bronchus and the patient was presented with minimal respiratory distress and the airway was secured with 4.5 $\mathrm{mm}$ tracheostomy tube after getting X-ray done.

Junction between the neck plate and the tube is the most common fractured site followed by the distal end and fenestra [2]. The possible reasons for the fracture are the usage of the same tracheostomy tube, alkaline bronchial secretions, repeated cleaning and sterilization, ageing of the tracheostomy tube, tissue reaction to the tube and manufacturing defects [5-10]. Tracheostomy tube fracture is more common between days 5 and 22 years and the duration of symptoms before the diagnosis is 1 day to 132 months [13]. Patient presents most commonly with mild respiratory distress [14]. Other symptoms include cough, haemoptysis, wheezing, recurrent pneumonia etc. In the paediatric population, death is also reported due to the small calibre of the airway $[15,16]$. Chest $X$-ray can easily diagnose the condition as the tube is radiopaque [13]. In our case, fracture occurred at the junction of the neck plate and tube as the tube was new so the likely reason could be the manufacturing defect and the likely reason for distal migration of the tube was the small size of the tube compared to the age, as the patient was on decannulation trial. 
Rigid bronchoscopy is the treatment of choice for fractured tracheostomy tube removal and it is done through the stoma site. Some authors also reported the need for thoracotomy and bronchotomy [16]. However, in our case, rigid bronchoscopy was done and the foreign body was removed through the oral cavity, instead of the stoma site, with a small-sized 4.5-mm tracheostomy tube in situ for ventilation, as the tube was of polyvinyl chloride material and thus pliable.

\section{Conclusions}

Tracheostomy tube fracture and migration is an avoidable complication. It can be avoided by careful inspection of the tracheostomy tube before changing the tube and appropriate tracheostomy care.

\section{Acknowledgements}

Not applicable

\section{Authors' contributions}

GS and SGoit wrote the case report. PK edited it. SG performed surgery under guidance of PK. AP edited photographs. All authors read and approved the final manuscript.

\section{Funding}

Not applicable

\section{Availability of data and materials}

Not applicable

\section{Declarations}

Ethics approval and consent to participate

Not applicable

\section{Consent for publication}

Written informed consent has been obtained from the parent of the patient.

\section{Competing interests}

The authors declare that they have no competing interests.

Received: 30 September 2021 Accepted: 31 December 2021

Published online: 23 February 2022

\section{References}

1. Okafor BC (1983) Fracture of tracheostomy tubes. Pathogenesis and prevention. J Laryngol Otol 97(8):771-774

2. Piromchai $P$, Lertchanaruengrit $P$, Vatanasapt $P$, Ratanaanekchai $T$, Thanaviratananich S (2010) Fractured metallic tracheostomy tube in a child: a case report and review of the literature. J Med Case Rep 4:234

3. Everitt E (2016) Care of patients with permanent tracheostomy. Nurs Time 112(21-3):20-22

4. Lewis CW, Carron JD, Perkins JA, Sie KC, Feudtner C (2003) Tracheostomy in paediatric patients: a national perspective. Arch Otolaryngol Head Neck Surg 129(5):523-529

5. Majid AA (1989) Fractured silver tracheostomy tube: a case report and literature review. Singapore Med J 30(6):602-604

6. Gana PN, Takwingi YM (2000) Fractured tacheostomy tubes in the tracheobronchial tree of a child. Int Paediatr Otorhinolaryngol 53(1):45-48

7. Parida PK, Kalaiarasi R, Gopalakrishnan S, Saxena SK (2014) Fractured and migrated tracheopstomy tube in the tracheobronchial tree. Int Paediatr Otorhinolaryngol 78(9):1472-1475
8. Krempl GA, Otto RA (1999) Fracture at fenestration of synthetic trachesostomy tube resulting in a tracheobronchial airway foreign body. South Med J 92(5):526-528

9. Kakar PK, Saharia PS (1972) An unusal foreign body in the trachea-bronchial tree. J Laryngol Otol 86(11):1155-1157

10. Kemper BI, Rosica N, Myers EN, Sparkman T (1972) Inner migration of the inner cannula: an unusual foreign body. Eye Ear Nose Throat Mon 51(7):257-258

11. Bassoe HH, Boe J (1960) Broken tracheostomy tube as a foreign body. Lancet 1(7132):1006-1007

12. Parida PK, Kalaiarasi R, Alexander A, Saxena SK (2020) Factors associated with fracture and migration of tracheostomy tube into trachea in children: A case series. Iran J Otolaryngol 32(6):379-383

13. So-ngern A, Boonsarngsuk V (2016) Fractured metallic tracheostomy tube: A rare complication of tracheostomy. Respir Med Case Rep 19:46-48

14. Jensen OV, Pedersen U (1988) Fracture in polyvinyl chloride tracheostomy tubes. J Laryngol Otol 102(4):380-381

15. Brockhurt PJ, Feltoe CK (1991) Corrosion and fracture of a silver tracheostomy tube. J Laryngol Otol 105(1):48-49

16. Ranjan K, Phookan J, Devi HR, Das MP (2016) Broken synthetic tracheostomy tube presenting as tracheobronchial foreign body- A case report. IOSR J Dental Med Sci 1(15):13-14

\section{Publisher's Note}

Springer Nature remains neutral with regard to jurisdictional claims in published maps and institutional affiliations.

\section{Submit your manuscript to a SpringerOpen ${ }^{\circ}$ journal and benefit from:}

- Convenient online submission

- Rigorous peer review

- Open access: articles freely available online

- High visibility within the field

Retaining the copyright to your article

Submit your next manuscript at springeropen.com 